\title{
Recherche de polluants inorganiques sur un ancien site de décharge d'ordures ménagères érigé en aire de jeux : cas de Bè-Aveto à Lomé au Togo
}

\author{
Ogouvidé AKPAKI ${ }^{1 *}$, Edem K. KOLEDZI ${ }^{1}$, Kwamivi N. SEGBEAYA ${ }^{2}$, Gnon BABA $^{2}$, \\ Koffi KILI $^{1}$ et Gado TCHANGBEDJI ${ }^{1}$ \\ ${ }^{1}$ Laboratoire de Gestion, Traitement et Valorisation des Déchets (GTVD), \\ Faculté des Sciences, Université de Lomé ; BP 1515 Lomé-Togo. \\ ${ }^{2}$ Laboratoire d'Assainissement Sciences de l'Eau et Environnement (LASEE), Faculté des Sciences et \\ Technique, Université de Kara ; B.P 404 Kara-Togo. \\ *Auteur correspondant ; E-mail: oakpaki@gmail.com, Tél :00228-90 325952
}

\section{RESUME}

Les déchets ménagers des pays en développement ne connaissent pas en général des tris. Les poubelles des ménages reçoivent les déchets de tout genre et il en est de même des décharges publiques qui sont par voie de conséquence potentiellement polluées et polluantes. Ce travail porte sur l'évaluation du degré de pollution de l'ancien site de décharge des ordures ménagères de Bè-Aveto à Lomé (Togo) actuellement érigé en une aire de jeux, en recherchant les teneurs des polluants chimiques. Ainsi, après avoir réalisé un zonage de l'aire de jeux, des échantillons représentatifs de sable ont été prélevés et analysés. Les résultats des analyses montrent que, l'actuelle aire de jeux renferme des éléments traces métalliques (ETM) tels que le plomb, le cadmium, l'arsenic, le mercure, de même que les nitrates et les phosphates dont les teneurs sont parfois plus de deux fois supérieures aux normes. Le taux de certains métaux comme le plomb laisse penser à d'autres sources telles que les pratiques cérémoniales au cours desquelles ce métal est souvent utilisé. En effet, le site ayant été auparavant une forêt sacrée, a pu recevoir des balles en plomb pour célébrer les dieux de la chasse. Toutefois, le taux de matière organique a été évalué afin de pouvoir postuler sur le potentiel de rétention des polluants organiques par ladite matière organique. Cette aire de jeux est donc polluée et nécessite des actions de dépollution. En marge des approches de dépollution chimiques proposées, il est aussi possible de garnir le site en gazon afin de réduire les risques de contamination.

(C) 2014 International Formulae Group. All rights reserved.

Mots clés : Déchet, décharge, jeux, polluant, contamination, site pollué, ETM.

\section{INTRODUCTION}

La pollution d'un site résulte de la présence sur ce site de substances polluantes imputables à des activités humaines et dont les caractéristiques sont de natures à générer des nuisances et des risques vis-à-vis des personnes, de l'environnement ou des biens (Hriz Ichrak et al., 2010). Les anciens sites de dépotoirs de déchets sont des réservoirs de polluants susceptibles d'affecter les écosystèmes, puis la chaîne alimentaire si des mesures de protection ou des travaux de dépollution ne sont pas réalisés avant la transformation des sites pour d'autres usages (Akcay et al., 2003). En effet, la pollution due au dépôt des déchets peut stériliser durablement les sols pour certains usages: habitations, espaces récréatifs. Aujourd'hui, les sols des sites de décharges, en activité ou non, font l'objet d'une attention particulière afin de réduire l'impact négatif sur l'environnement. 
C'est le cas de l'ancien site de décharge de Bè-Aveto au cœur de la ville de Lomé (Photo 1), aujourd'hui devenu une aire de jeux (Photo 2). Ce site a servi de décharge intermédiaire pendant des années pour les ordures ménagères de l'agglomération de Lomé. Aujourd'hui, ce site est transformé en air de jeu sans aucune action de dépollution en amont. Ce site étant situé à proximité de l'océan atlantique, le transfert de polluants vers la nappe phréatique est à craindre.

Ainsi, le sujet de cette étude porte sur la recherche des teneurs des polluants inorganiques notamment les métaux lourds, les nitrates et les phosphates dans les horizons de surface de ce site. Cette recherche permettra alors d'évaluer l'état de pollution chimique du site et proposer une solution de dépollution. Les métaux lourds retenus dans cette étude sont le cadmium, le plomb, le mercure et l'arsenic en raison de leur toxicité. En complément à ces métaux, les teneurs en matière organique, en nitrates, et en phosphates sont déterminés. Les quantités de ces polluants devront servir d'indicateurs de l'état de pollution du site et le risque encouru par les riverains et les usagers.

\section{MATERIEL ET METHODES}

Echantillonnage et traitement des données

Pour obtenir des échantillons assez représentatifs, un zonage du site a été réalisé. Ainsi, huit zones ont été définies avec des surfaces proportionnelles à la concentration des déchets restés enfouis dans le sol (Figure 1). Les échantillons prélevés sont les sols des premiers horizons du site. Les zones A et B, ont chacune une superficie d'environ $105 \mathrm{~m}^{2}$ et 35 échantillons y ont été prélevés. Les zones $\mathrm{C}, \mathrm{D}, \mathrm{E}$ et $\mathrm{F}$ ont une superficie de $75 \mathrm{~m}^{2}$ chacune avec 25 échantillons prélevés. Les zones $\mathrm{G}$ et $\mathrm{H}$ ont une superficie de $45 \mathrm{~m}^{2}$ chacune et 15 échantillons prélevés. Au total deux cents échantillons ont été prélevés.

$\mathrm{Au}$ laboratoire, les échantillons sont séchés à la température ambiante, $35^{\circ} \mathrm{C}$ environ, durant 3 à 4 heures environ pour réduire l'humidité, puis broyés dans un mortier en porcelaine et tamisés à l'aide d'un tamis de $4 \mathrm{~mm}$ de diamètre. Ces opérations ont été faites avec précaution pour éviter des éventuelles contaminations. Pour les analyses, deux échantillons composites sont reconstitués par zone en mélangeant et en homogénéisant deux grammes de chaque échantillon. Ainsi, 16 échantillons composites notés respectivement $\left(\mathrm{A}_{\mathrm{I}}, \mathrm{A}_{\mathrm{II}}, \mathrm{B}_{\mathrm{I}}, \mathrm{B}_{\mathrm{II}}, \mathrm{C}_{\mathrm{I}}, \mathrm{C}_{\mathrm{II}}\right.$,

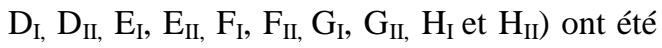
utilisés pour les analyses. Pour le dosage de l'arsenic (As), du mercure (Hg) et des phosphates, à cause du coût élevé des analyses, seuls deux échantillons composites sont reconstitués en regroupant les zones $\mathrm{A}$, $\mathrm{B}, \mathrm{C}$ et $\mathrm{D}$ puis les zones $\mathrm{E}, \mathrm{F}, \mathrm{G}$ et $\mathrm{H}$ pour réduire le nombre des analyses.

Analyses des polluants

Dosage des métaux lourds $(\mathrm{Cd}, \mathrm{Pb}, \mathrm{Hg}$ et As)

Minéralisation des échantillons: Le dosage des métaux dans les échantillons nécessite une phase de minéralisation. Elle est réalisée à l'aide d'un mélange de solutions concentrées d'acide nitrique $\left(\mathrm{HNO}_{3}\right)$, d'acide chlorhydrique $(\mathrm{HCl})$ et d'eau oxygénée $\left(\mathrm{H}_{2} \mathrm{O}_{2}\right)$. Ainsi, à $0,5 \mathrm{~g}$ d'échantillon finement broyé et légèrement humidifié avec de l'eau distillée, est ajouté respectivement $5 \mathrm{ml}$ d'eau oxygénée, $5 \mathrm{ml}$ d'acide nitrique à $68 \%$ et 15 ml d'acide chlorhydrique à $37 \%$.

Le mélange est chauffé au bain de sable à une température d'environ $150{ }^{\circ} \mathrm{C}$ pendant 2 heures. Le résidu solide est récupéré avec $2 \mathrm{ml}$ $\mathrm{d}^{\prime} \mathrm{HNO}_{3}$ à $10 \%$ puis porté à nouveau à ébullition pendant quelques minutes pour obtenir la dissolution complète.

Après refroidissement, le minéralisât est ramené à $100 \mathrm{ml}$ avec de l'eau distillée, puis filtré à l'aide du papier Wattman. Les solutions ainsi obtenues sont conservées à une température de $4{ }^{\circ} \mathrm{C}$. Le dosage des éléments métalliques est réalisé à partir de ce filtrât à l'aide d'un spectrophotomètre d'absorption atomique (mode flamme : Aanalyst 800 (PERKIN ELMER)) (Adouby et al., 2007 ; Bustamante et al., 2008; Belyaeva and Haynes, 2009 ; Koffi et al., 2010).

\section{Dosage des nitrates}

A $10 \mathrm{~g}$ de sol frais placé dans une bouteille à agitation de $100 \mathrm{ml}$, ont été ajoutés $20 \mathrm{ml}$ de solution de sulfate de potassium à 0,5 mol/l. La bouteille fermée est mise en agitation pendant 30 minutes. $10 \mathrm{ml}$ de la solution d'hydroxyde de sodium à $0,4 \mathrm{~mol} / \mathrm{l}$ 
ont été ensuite ajoutés au mélange toujours sous agitation pendant une 1 heure pour favoriser le développement d'une coloration jaune. Si cette coloration reste stable après 12 heures, la mesure de la concentration des nitrates est effectuée par spectrophotométrie à 410 nm (Alasac, 2007 ; Mathieu et Pieltain, 2003).

\section{Dosage des phosphates}

Pour le dosage des phosphates, nous avons utilisé la méthode de l'analyse colorimétrique du phosphore. L'analyse des phosphates par la méthode colorimétrique du phosphore est effectuée sur les mêmes minéralisâts qui ont servi aux dosages du cadmium et du plomb. Toutes les analyses du phosphore ont été faites selon la méthode de Murphy et Riley qui est basée sur la formation du bleu de molybdène dans la solution d'acide (Mathieu et Pieltain, 2003).

\section{Evaluation de la matière organique}

La matière organique est évaluée par la méthode de WALKLEY et de BLACK (Mathieu et Pieltain, 2003). Le principe est basé sur l'oxydation de la matière organique par un excès de dichromate en milieu acide (Tumuhairwe et al., 2009 ; Waskman, 1936).

Méthode de calcul du taux de COT et de la matière organique (MO)

- $588 \mathrm{~g}(2 \mathrm{~mol})$ de $\mathrm{K}_{2} \mathrm{Cr}_{2} \mathrm{O}_{7}$ correspondent à $36 \mathrm{~g}$ (3 mol) de carbone.

- $1 \mathrm{ml}$ de solution de bichromate de potassium oxyde $(3 \div 1000)=0,0003 \mathrm{~g}$ ou 3 milligramme de carbone.

Expérimentalement, il a été constaté que l'oxydation du carbone par cette méthode ne se fait qu'à $77 \%$ de sa totalité : il faut corriger le résultat par le facteur $(100 \div 77)=$ 1,2987. Ce qui nous amène à dire que : $1 \mathrm{ml}$ de solution de bichromate de potassium correspond à $(3 \mathrm{mg} \times 12987)=3,8961$ ou 3,9 mg de carbone par excès.

\section{RESULTATS}

La recherche des polluants chimiques sur l'ancien site de décharge des ordures ménagères de Bè-Aveto amène à effectuer des analyses chimiques dont les résultats se présentent ainsi :

\section{Teneur des métaux lourds}

L'historique du site et de la composition des ordures ménagères suscitent la recherche des métaux lourds ayant une toxicité avérée pour l'homme. Ainsi, quatre éléments toxiques notamment le cadmium, le plomb, le mercure et l'arsenic sont retenus dans cette étude. Le Tableau 1, présente les teneurs des éléments arsenic et mercure. Le Tableau 2, présente les teneurs du cadmium, comprise entre $5,92 \mathrm{ppm}$ et $37,28 \mathrm{ppm}$ et celles du plomb, varient entre $4,38 \mathrm{ppm}$ et 108,52 ppm.

\section{Teneur des nitrates et des phosphates}

Les teneurs des nitrates présentées dans le Tableau 3, sont comprises entre $1.52 \mathrm{ppm}$ et $60.62 \mathrm{ppm}$. Et le Tableau 4, présente les teneurs des phosphates qui varient entre $0.05 \%$ et $0.47 \%$.

\section{Taux de carbone organique total}

Le Tableau 5 présente les teneurs en matière organique (MO) et en masse de carbone $\left(\mathrm{M}_{\mathrm{C}}\right)$ dans les différents échantillons composites. Ces teneurs varient entre $1.12 \%$ et $2.79 \%$ et dont leur moyenne est $1.96 \%$. Ces résultats montrent que le site est riche en MO.

\section{Analyse statistique descriptive des résultats \\ Pour disposer d'un minimum} d'information sur les coefficients de variation des teneurs des polluants, nous avons procédé à une analyse statistique descriptive de certains polluants : cadmium, plomb, nitrates et phosphates et de la matière organique afin de voir leur répartition sur l'ensemble du site. Ces données sont présentées dans le Tableau 6.

\section{Teneur en cadmium et en plomb}

Dans les sols non contaminés, la teneur minimale du cadmium est de 13 ppm et celui de plomb est de 16 ppm (Hriz Ichrak et al., 2010). Les sols du site d'étude ont des teneurs minimales de 5,92 ppm pour le cadmium et $4,38 \mathrm{ppm}$ pour le plomb. Les teneurs moyennes sont de 20,53 ppm pour les concentrations du cadmium et $46,39 \mathrm{ppm}$ pour le plomb. Ces teneurs étant largement supérieures aux valeurs minimales dans les sols non contaminés, on en déduit que le site 
de Bè-Aveto est très pollué en cadmium et en plomb.

La pollution en cadmium et en plomb est hétérogène sur l'ensemble du site car les coefficients de variations (CV), $44 \%$ pour le cadmium et $61 \%$ pour le plomb sont supérieurs à la moyenne qui est 33\%. La dispersion de ces polluants reflète l'état d'un site de dépotoir d'ordures.

\section{Teneur en nitrates}

La teneur du site en nitrates est aussi hétérogène avec un coefficient de variation $81 \%$ très élevé par rapport $33 \%$ qui est la moyenne, ce qui confirme que la pollution par les nitrates est purement de nature diffuse.

En comparant les valeurs minimale 1,52 ppm, maximale 60,62 ppm et la moyenne 20,88 ppm en nitrates aux valeurs guides du domaine acidogénèse et méthanogénèse des sols des dépotoirs des ordures ménagères qui sont comprises entre 4,425 ppm et 44,248 ppm (Alasac, 2007), il apparaît que presque l'ensemble du sol est acide mais que cette acidité est hétérogène au vu du coefficient de variation qui est $81 \%$.

\section{Teneur en phosphate}

En faisant un parallèle entre les pourcentages minimal $0,05 \%$, maximal $0,47 \%$ et la moyenne $0,23 \%$ en phosphates avec valeurs guides du domaine acidogénèse et méthanogénèse des sols des dépotoirs des ordures ménagères qui sont comprises entre $0,0001 \%$ et $0,0153 \%$ (Mathieu et Pieltain, 2003), on peut estimer que l'ensemble du site est très acide. De même, cette acidité est hétérogène sur l'ensemble de l'espace considéré en tenant compte du coefficient de variation qui est $52 \%$.

\section{Teneur en matière organique}

Le taux de la matière organique est évalué afin d'estimer la capacité de rétention des polluants organiques du site. Ce taux évolue entre $1,12 \%$ et $2,79 \%$. Comparé à la valeur guide ( $1 \%$ à $2 \%$ ) admise pour les sols minéralisés des sites d'anciens dépotoirs d'ordures ménagères, on peut suspecter une accumulation de polluants organiques dans les sols du site de Bè-Aveto (Tumuhairwe et al., 2009).

Le coefficient de répartition de la matière organique calculé dans les sols du site est égal à $27 \%$ et est inférieur à la moyenne de $33 \%$. Il traduit alors une répartition très homogène de la matière organique dans les sols de surface du site. Cette répartition homogène peut s'expliquer par les dépôts successifs aléatoires mais homogènes des déchets sur le site non aménagé et par une infiltration homogène de la matière organique issue du lessivage des déchets.

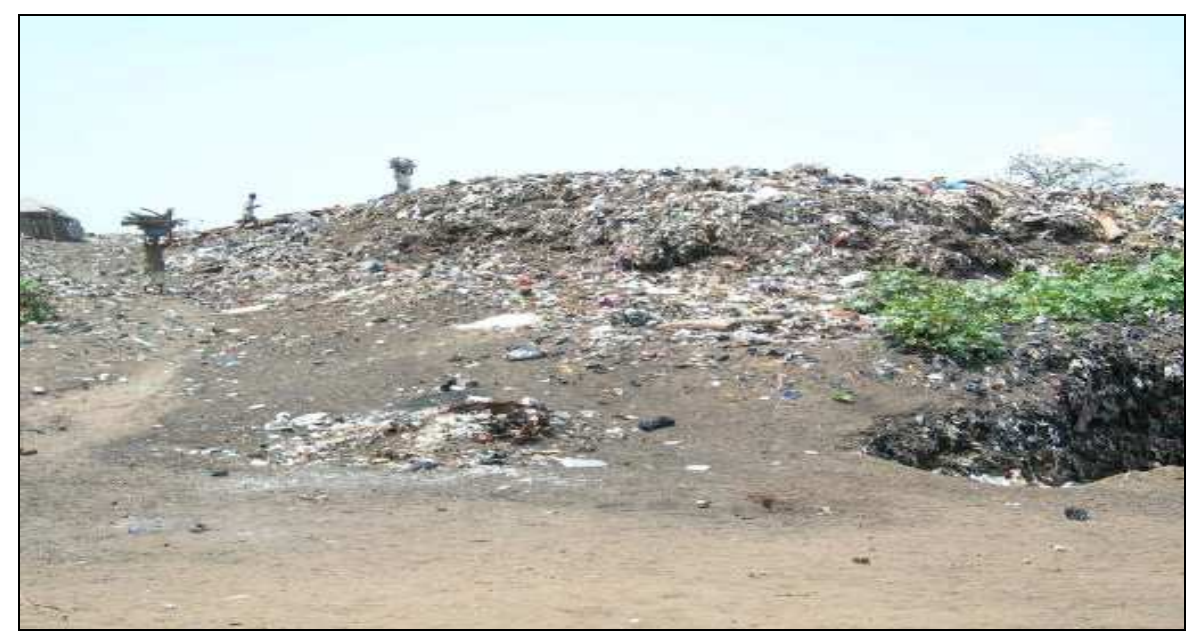

Photo 1: Montagne des ordures ménagères du site (21/10/2006). 




Photo 2 : Site devenu une aire de jeu (21/08/2008).

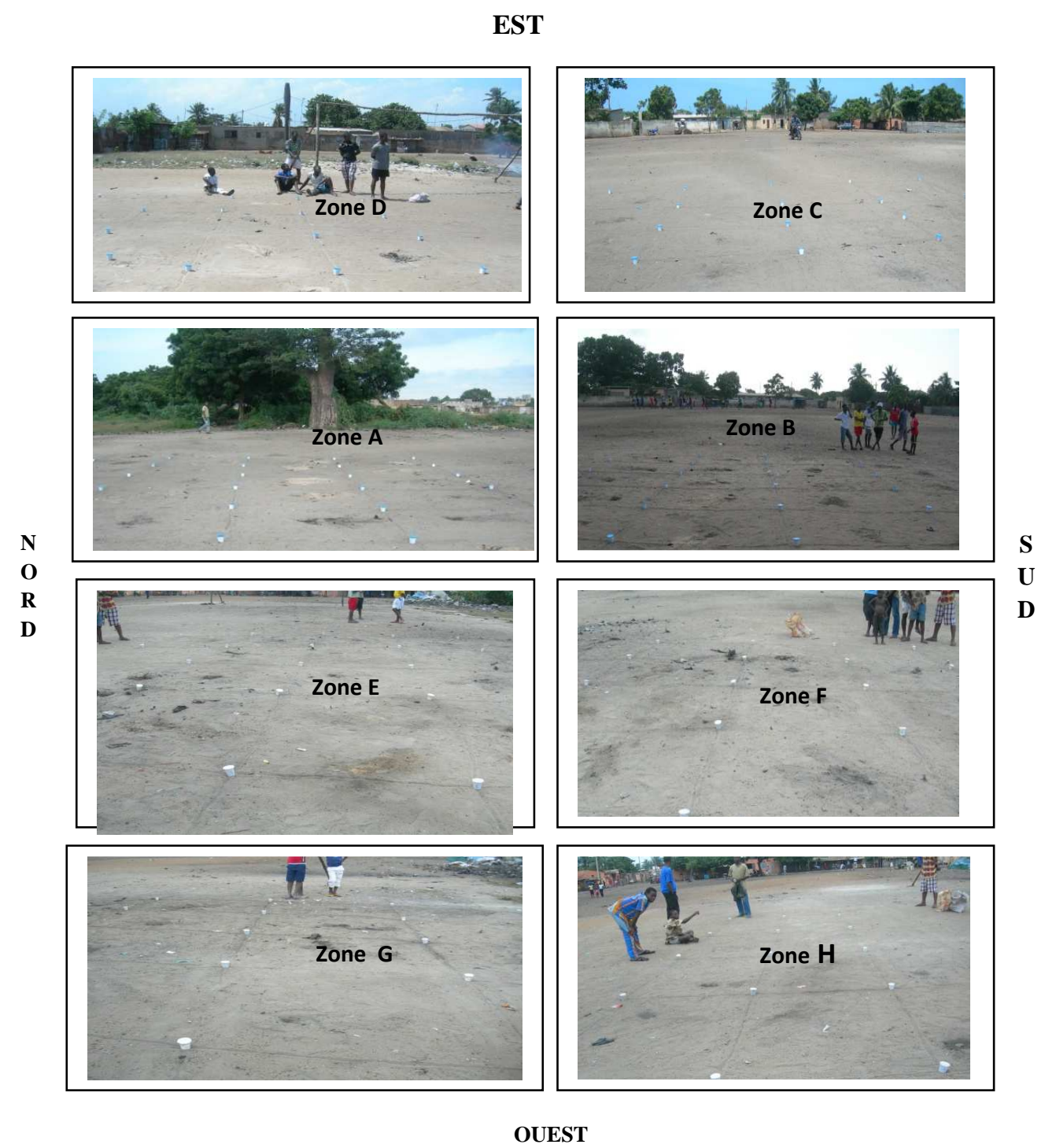

Figure 1 : Délimitation et choix des zones de prélèvement des échantillons. 
Tableau 1 : Teneur en arsenic et en mercure.

\begin{tabular}{lcc}
\hline Eléments & Echantillon 1 & Echantillon 2 \\
\hline Arsenic & Trace $\mathrm{C}<0,2 \mu \mathrm{g} / \mathrm{l}$ & Trace $\mathrm{C}<0,2 \mu \mathrm{g} / \mathrm{l}$ \\
Mercure & $0,0122 \mathrm{mg} / \mathrm{kg}$ & $0,0228 \mathrm{mg} / \mathrm{kg}$ \\
\hline
\end{tabular}

Tableau 2 : Teneur en cadmium et en plomb.

\begin{tabular}{lcc}
\hline Echantillons composites & Cadmium $(\mathbf{p p m})$ & Plomb $(\mathbf{p p m})$ \\
\hline $\mathrm{A}_{\mathrm{I}}$ & 29,66 & 74,82 \\
$\mathrm{~A}_{\mathrm{II}}$ & 31,08 & 59,16 \\
$\mathrm{~B}_{\mathrm{I}}$ & 09,28 & 19,94 \\
$\mathrm{~B}_{\mathrm{II}}$ & 16,12 & 39,98 \\
$\mathrm{C}_{\mathrm{I}}$ & 05,92 & 04,38 \\
$\mathrm{C}_{\mathrm{II}}$ & 12,00 & 09,04 \\
$\mathrm{D}_{\mathrm{I}}$ & 18,50 & 44,92 \\
$\mathrm{D}_{\mathrm{II}}$ & 15,38 & 56,86 \\
$\mathrm{E}_{\mathrm{I}}$ & 27,88 & 108,52 \\
$\mathrm{E}_{\mathrm{II}}$ & 27,08 & 59,70 \\
$\mathrm{~F}_{\mathrm{I}}$ & 23,00 & 62,24 \\
$\mathrm{~F}_{\mathrm{II}}$ & 28,92 & 79,42 \\
$\mathrm{G}_{\mathrm{I}}$ & 25,98 & 34,30 \\
$\mathrm{G}_{\mathrm{II}}$ & 37,28 & 62,40 \\
$\mathrm{H}_{\mathrm{I}}$ & 09,34 & 12,94 \\
$\mathrm{H}_{\mathrm{II}}$ & 11,08 & 13,72 \\
\hline
\end{tabular}

Tableau 3 : Teneur en nitrates.

\begin{tabular}{lc}
\hline Echantillon & Concentration (ppm) \\
\hline $\mathrm{A}_{\mathrm{I}}$ & 17,556 \\
$\mathrm{~A}_{\mathrm{II}}$ & 7,142 \\
$\mathrm{~B}_{\mathrm{I}}$ & 3,622 \\
$\mathrm{~B}_{\text {II }}$ & 1,522 \\
$\mathrm{C}_{\mathrm{I}}$ & 2,190 \\
$\mathrm{C}_{\text {II }}$ & 9,810 \\
$\mathrm{D}_{\mathrm{I}}$ & 37,836 \\
$\mathrm{D}_{\text {II }}$ & 13,048 \\
$\mathrm{E}_{\mathrm{I}}$ & 26,396 \\
$\mathrm{E}_{\mathrm{II}}$ & 22,896 \\
$\mathrm{~F}_{\mathrm{I}}$ & 15,328 \\
$\mathrm{~F}_{\text {II }}$ & 36,990 \\
$\mathrm{G}_{\mathrm{I}}$ & 30,636 \\
$\mathrm{G}_{\text {II }}$ & 2,786 \\
$\mathrm{H}_{\mathrm{I}}$ & 60,616 \\
$\mathrm{H}_{\text {II }}$ & 45,796 \\
\hline
\end{tabular}


Tableau 4 : Teneur en phosphate organique.

\begin{tabular}{lccc}
\hline Echantillon & $\begin{array}{c}\text { Concentration } \\
\text { (ppm) } \mathbf{P O}_{\mathbf{4}}{ }^{3-}\end{array}$ & \multicolumn{2}{c}{ Pourcentage (\%) } \\
\cline { 3 - 4 } & 4729 & 0,4729 & 1,0829 \\
\hline $\mathrm{A}_{\mathrm{I}}$ & 548 & 0,0548 & 0,1255 \\
$\mathrm{~A}_{\mathrm{II}}$ & 3395 & 0,3395 & 0,7774 \\
$\mathrm{~B}_{\mathrm{I}}$ & 1999 & 0,1999 & 0,4577 \\
$\mathrm{~B}_{\mathrm{II}}$ & 2150 & 0,2150 & 0,4923 \\
$\mathrm{C}_{\mathrm{I}}$ & 2581 & 0,2581 & 0,5910 \\
$\mathrm{C}_{\mathrm{II}}$ & 1232 & 0,1232 & 0,2821 \\
$\mathrm{D}_{\mathrm{I}}$ & 1177 & 0,1177 & 0,2669 \\
$\mathrm{D}_{\mathrm{II}}$ & 2013 & 0,2013 & 0,4609 \\
$\mathrm{E}_{\mathrm{I}}$ & 900 & 0,0900 & 0,2061 \\
$\mathrm{E}_{\mathrm{II}}$ & 3324 & 0,3324 & 0,7612 \\
$\mathrm{~F}_{\mathrm{I}}$ & 2937 & 0,2937 & 0,6725 \\
$\mathrm{~F}_{\mathrm{II}}$ & 3571 & 0,3571 & 0,8177 \\
$\mathrm{G}_{\mathrm{I}}$ & 4277 & 0,4277 & 0,9794 \\
$\mathrm{G}_{\mathrm{II}}$ & 1106 & 0,1106 & 0,2532 \\
$\mathrm{H}_{\mathrm{I}}$ & 1204 & 0,1204 & 0,2757 \\
$\mathrm{H}_{\mathrm{II}}$ & & & \\
\hline
\end{tabular}

Tableau 5 : Teneur du carbone et de la matière organique.

\begin{tabular}{lcc}
\hline Echantillon & $\mathbf{M}_{\mathbf{c}}(\boldsymbol{\%} \mathbf{C})$ & $\mathbf{\% M O}$ \\
\hline $\mathrm{A}_{\mathrm{I}}$ & 1,53 & 2,64 \\
$\mathrm{~A}_{\mathrm{II}}$ & 1,62 & 2,79 \\
$\mathrm{~B}_{\mathrm{I}}$ & 1,10 & 1,89 \\
$\mathrm{~B}_{\text {II }}$ & 1,07 & 1,85 \\
$\mathrm{C}_{\mathrm{I}}$ & 0,78 & 1,34 \\
$\mathrm{C}_{\mathrm{II}}$ & 0,82 & 1,41 \\
$\mathrm{D}_{\mathrm{I}}$ & 0,65 & 1,12 \\
$\mathrm{D}_{\text {II }}$ & 1,23 & 2,12 \\
$\mathrm{E}_{\mathrm{I}}$ & 1,19 & 2,06 \\
$\mathrm{E}_{\mathrm{II}}$ & 1,43 & 2,48 \\
$\mathrm{~F}_{\mathrm{I}}$ & 1,23 & 2,12 \\
$\mathrm{~F}_{\mathrm{II}}$ & 1,39 & 2,40 \\
$\mathrm{G}_{\mathrm{I}}$ & 1,41 & 2,44 \\
$\mathrm{G}_{\mathrm{II}}$ & 1,35 & 2,34 \\
$\mathrm{H}_{\mathrm{I}}$ & 0,68 & 119 \\
$\mathrm{H}_{\text {II }}$ & 0,71 & 1,24 \\
\hline \multicolumn{2}{c}{$:$ effectif $;$} & $\mathbf{C V}:$ coefficient de variation (en \%)
\end{tabular}


Tableau 6 : Analyse statistique descriptive des teneurs.

\begin{tabular}{lccccccc}
\hline \multicolumn{9}{c}{ Statistiques descriptives } & & & NORMES \\
\hline & N & Minimum & Maximum & Moyenne & $\begin{array}{c}\text { Ecart } \\
\text { type }\end{array}$ & CV & Valeurs limites \\
\hline $\begin{array}{l}\text { Cadmium } \\
(\mathrm{ppm})\end{array}$ & 16 & 5,92 & 37,28 & 20,53 & 9,20 & 44 & 0,2 \\
$\begin{array}{l}\text { Plomb } \\
(\mathrm{ppm})\end{array}$ & 16 & 4,38 & 108,52 & 46,39 & 28,45 & 61 & $13-16$ \\
$\begin{array}{l}\text { Nitrates } \\
(\mathrm{ppm})\end{array}$ & 16 & 1,52 & 60,62 & 20,88 & 17,02 & 81 & $4,125-44,248$ \\
$\begin{array}{l}\text { Phosphates } \\
(\%)\end{array}$ & 16 & 0,05 & 0,47 & 0,23 & 0,12 & 52 & $0,0001-0,0153$ \\
MO (\%) & 16 & 1,12 & 2,79 & 1,96 & 0,53 & 27 & $1-2$ \\
\hline$*$ IFE, 2006; Mathieu et Pieltain, 2003 & & & & & & \\
\end{tabular}

\section{DISCUSSION}

L'analyse des différents résultats montre que le site étudié regroupe plusieurs polluants chimiques. Il apparaît aussi que leur répartition n'est pas uniforme puisque d'une région à l'autre, on ne retrouve pas les concentrations semblables.

\section{Teneur en cadmium}

La teneur en cadmium du site dont présente le Tableau 2, est comprise entre 05,92 et $37,28 \mathrm{ppm}$. Comparée à la valeur minimale dans les sols non contaminés fixée à $0,2 \mathrm{ppm}$ par la norme européenne, on en déduit que le site est fortement pollué (IFE, 2006; Yobouet et al., 2010). Cette forte accumulation du cadmium peut s'expliquer par la composition et la nature des ordures ménagères. En effet, les ordures ménagère, renferment des quantités importantes de piles où accumulateurs usagés de type $\mathrm{Ni}-\mathrm{Cd}$, des pigments, des pneus usés qui contiennent du cadmium et des verres. La forte proportion des piles usagées dans les ordures ménagères qui ont été déversées sur le site peut permettre d'avancer l'hypothèse d'une utilisation courante des piles par les ménages des quartiers riverains du site. En effet, pendant très longtemps, le taux d'électrification des ménages riverains au site était très faible et l'usage des piles et des accumulateurs est très fréquent pour répondre aux besoins en énergie électrique. Une autre explication plausible de la forte concentration du cadmium dans les sols du site, est l'incinération fréquente des déchets déposés sur le site. Le cadmium libéré lors de ces incinérations peut être ensuite entraîné par lessivage vers le sol.

\section{Teneur en plomb}

La teneur en plomb du site est comprise entre 04,38 et $108,52 \mathrm{ppm}$. Comparée la norme européenne (française) qui fixe la concentration du plomb à une valeur comprise entre $13 \mathrm{ppm}$ et $16 \mathrm{ppm}$ (valeur seuil) (IFE, 2006), on en déduit que les sols du site sont contaminés par le plomb. Les hypothèses suivantes peuvent être avancées pour expliquer cette forte accumulation du plomb sur le site.

Le site aurait reçu pendant très longtemps des déchets tels que les piles et les accumulateurs usagés à base de plomb, les produits de vidanges des moteurs d'automobiles, les résidus de produits utilisés dans la soudure des métaux, et les déchets issus des appareils électroménagers notamment les écrans de téléviseur contenant tous du plomb. Plus encore, le site étant une ancienne forêt sacrée très dense, son cycle biogéochimique peut contribuer à l'accumulation du plomb dans le sol. Le caractère sacré de cette forêt dédiée aux dieux de la foudre a certainement occasionné des rituels et cérémonies traditionnelles au cours desquelles des objets sacrés tels que les 
munitions et autres objets fabriquées à bases du plomb seraient abondamment utilisés. Le site aurait subi une incinération très poussée libérant le plomb contenu dans les fractions non biodégradables des déchets. Le lessivage des déchets et l'érosion en surface sont également des facteurs favorables au transfert du plomb vers le sol.

\section{Teneur en arsenic}

Dans les deux échantillons analysés, la teneur en arsenic est inférieure à $0,04 \mathrm{mg} / \mathrm{kg}$. En comparant cette teneur à la norme européenne (Pays-Bas) fixée entre $29 \mathrm{mg} / \mathrm{kg}$ et $55 \mathrm{mg} / \mathrm{kg}$ (Mathieu et Pieltain, 2003), on peut, dans une première approximation, considérer que le site de Bè-Aveto n'est pas contaminé par l'arsenic. Toutefois, étant donné que les analyses ont été réalisées sur des échantillons composites, on peut suspecter un phénomène de dilution par des échantillons renfermant des teneurs très faibles en arsenic. D'autre part, les hypothèses suivantes peuvent être avancées pour justifier la faible concentration de l'arsenic sur le site :

Les ordures ménagères reçues pendant très longtemps par le site ne contiennent pas assez de métal arsenic pour induire une pollution importante du site par ce métal. Il est possible qu'une grande partie de l'arsenic mobilisé dans les déchets est dissoute à travers les réactions chimiques complexes qui ont lieu à l'interface sol-déchet et est ensuite transporté par infiltration à travers le sol vers les eaux de la nappe phréatique. Enfin, l'hypothèse d'un usage peu courant d'objets contenant de l'arsenic peut être avancée.

\section{Teneur en mercure}

La teneur du mercure présentée dans le Tableau 1, évolue entre 0,0122 et 0,0228 $\mathrm{mg} / \mathrm{kg}$. Elle est largement inférieure à la norme européenne (Pays-Bas) comprise entre $0,3 \mathrm{mg} / \mathrm{kg}$ et $10 \mathrm{mg} / \mathrm{kg}$ et fixée comme valeur guide d'intervention de décontamination des sites pollués (Mathieu et Pieltain, 2003). Cette faible concentration peut s'expliquer par les mêmes hypothèses évoquées dans le cas de l'arsenic.

\section{Teneur en nitrates}

Les données de la littérature révèlent que la teneur en nitrates qui indique le domaine d'évolution des phases acidogénèse et méthanogénèse des sols des dépotoirs d'ordures ménagères est généralement dans la gamme de 4,425 ppm et 44,248 ppm (Alasac, 2007). Les valeurs retrouvées dans les échantillons des différentes zones, excepté celles des zones $\mathrm{B}_{\mathrm{I}}, \mathrm{B}_{\mathrm{II}}, \mathrm{C}_{\mathrm{I}}, \mathrm{G}_{\mathrm{II}}$ et $\mathrm{H}_{\mathrm{I}}$ sont dans cette gamme et montrent que globalement, la biodégradation dans les sols du site se trouve dans les phases acidogénèse et méthanogénèse avec une phase méthanogénèse plus prononcée. Ces valeurs confirment un site ancien de dépotoirs d'ordures ménagères d'âge moins avancé. Les faibles teneurs retrouvées dans les échantillons des zones $\mathrm{B}_{\mathrm{I}}$, $B_{\text {II }}, C_{I}$ et $G_{I I}$ et la teneur moyenne de 60,616 ppm dans les échantillons de la zone $\mathrm{H}_{\mathrm{I}}$ peuvent s'expliquer par un dépôt assez aléatoire des déchets sur le site. L'ensemble des teneurs montre également que les ordures ménagères que le site aurait reçu contiennent une quantité importante de matériaux organiques riches en azote.

\section{Teneur en phosphates}

Les teneurs obtenues sur l'ensemble des zones, comparées aux valeurs guides qui indiquent les domaines d'évolution des phases acidogénèse et méthanogénèse des sols des dépotoirs des ordures ménagères ( 1 ppm à 153 ppm) (Mathieu et Pieltain, 2003), confirment que la biodégradation dans les sols du site se trouve dans les phases acidogénèse et méthanogénèse. En considérant le cycle général du phosphore, on peut estimer qu'une quantité importante de matériaux organiques riches en phosphore serait contenue dans les ordures ménagères que le site aurait reçues.

\section{Teneur en matière organique (MO)}

La concentration du sol en matière organique peut se justifier par le fait que les matières fermentescibles représentent une part importante dans la composition physique des ordures ménagères. 
La couche de matière organique a une conséquence directe sur la capacité du sol à retenir les polluants. En effet, d'après la littérature, la teneur en matière organique est le paramètre qui présente une très bonne corrélation avec le pouvoir de rétention des polluants organiques et métalliques par le sol (Hargreaves et al., 2008). A titre d'exemple, il a été montré que pour une teneur en matière organique de $2 \%$, les sols ont la capacité de retenir $0,64 \mathrm{~L} / \mathrm{kg}$ de pesticide (Atrazine) et pour $4 \%$ de matière organique, ils peuvent retenir, $1,71 \mathrm{~L} / \mathrm{kg}$ de pesticide (Atrazine) (Barriuso, 1996). La teneur en matière organique des sols du site d'étude se situe au environs de $2 \%$. On peut alors prétendre que le site est potentiellement riche en polluants organiques.

D'un point de vue pédologique, la matière organique joue un rôle prépondérant dans la sorption de polluants organiques. Les phénomènes de sorption (terme qui regroupe l'adsorption, l'absorption et la désorption) interviennent notamment en termes de retard par rapport à l'écoulement de la solution du sol (Gueu et al., 2006 ; Yobouet et al., 2010).

Dans le sol, la présence de la matière organique, notamment de nature humique, est importante pour l'adsorption des polluants organiques. Par exemple, le $\mathrm{pH}$ du sol détermine entièrement l'adsorption: un $\mathrm{pH}$ élevé entraîne une grande quantité de charges négatives d'une substance polluante sur la matière organique, et pour un $\mathrm{pH}$ faible la charge positive d'une substance polluante est plus élevée (Hargreaves et al., 2008).

\section{Conclusion}

Pour un certain nombre de polluants, il existe des normes en dessous desquelles on considère que le polluant ne présente pas de risque pour l'environnement ni pour les humains. Ce risque dépendra non seulement de la toxicité du polluant, mais aussi de sa propension à s'accumuler dans les tissus biologiques (bioaccumulation) (Farrel et Jones, 2009 ; Lemière et al., 2008) . Cependant, l'importance, la consistance et le niveau de prise en considération des problèmes de sites contaminés varient énormément d'un pays à un autre. Nombreux sont encore les pays dont le Togo qui manquent d'informations sur ce sujet ou qui n'ont pas de législation adaptée, de moyens humains, techniques ou financiers pour faire face à ce problème.

Le présent travail a porté sur la recherche de certains polluants chimiques de l'ancien site de décharge des ordures ménagères de Bè-Aveto qui est érigé en aire de jeux pour la population environnante. Nous avons d'abord procédé à un zonage du site afin de situer les régions d'échantillonnage. Ensuite, les échantillons prélevés ont été soumis à une recherche qualitative et quantitative des polluants inorganiques. Toutefois, nous avons évalué le taux de matière organique de l'ensemble de l'aire de jeux. Ainsi, au terme de nos travaux, nous pouvons noter que :

- le site de Bè-Aveto est fortement pollué en cadmium, en plomb et très riche en mercure, mais ces pollutions sont plus ou moins hétérogènes sur l'ensemble du site. La teneur de ces métaux lourds dépasse les normes ;

- les teneurs en nitrates, et en phosphore sont très élevées et les valeurs ou les pourcentages guide d'acidogénèse et de méthanogénèse sont atteintes et voire dépassées par endroits, ce qui laisse supposer que le sol serait très acide ; - le site a une couche très riche en matière organique avec une répartition très homogène. Cette teneur en MO aurait comme pour conséquences la rétention des polluants organiques dont les activités urbaines en l'occurrence la gestion et le traitement des déchets sont l'une des sources principales génératrices des polluants organiques persistants.

Cette aire de jeux peut donc être considérée comme chargée en polluants chimiques. Et parmi les solutions d'approches formulées, il est à noter la garniture du site en gazon à défaut d'une décontamination chimique efficace. La végétation de cette aire pourrait limiter les contacts des usagers avec des polluants chimiques et réduire les contaminations humaines. 


\section{REFERENCES}

Akcay H, Oguz A, Karapire C. 2003. Study of heavy metal pollution speciation in Buyak Menderes and Gediz river sediments. Water Research, 37: 813-822.

Alasac N. 2007. Revue, dosage des métaux lourds (As, $\mathrm{Cd}, \mathrm{Cr}, \mathrm{Cu}, \mathrm{Ni}, \mathrm{Pb}, \mathrm{Zn}$ et $\mathrm{Hg}$ ) dans les sols. Annales de Toxicologie Analytique, 15(1).

Barriuso E, Eko O. M, Iglesias E, Houot S. 1996. Modification de la mobilité de pesticide dans les sols après addition de la matière exogène. $1^{\text {ère }}$ journée nationale de l'étude des sols, AEES, Rennes.

Belyaeva O. N, Haynes R. 2009. Chemical, microbial and physical properties of manufactured soils produced by cocomposting municipal green waste with coal fly ash. Bioresource Technology, 100: 5203-5209.

Ben HI, Mediouni H, Kacem M, Mgaidi A. 2010. Caractérisation de la mobilisation des polluants inorganiques dans des sols pollués. Actes du colloque international francophone E3D, 28-31 mars 2010, Alexandrie, Egypte.

Bustamante MA, Paredes C, Marhuenda-Egea FC, Perez-Espinosa A, Bernal MP, Moral R. 2008. Co-composting of distillery wastes with animal manures: Carbon and nitrogen transformations in the evaluation of compost stability. Chemosphere, 72: 551-557.

Farrel M, Jones DL. 2009. Critical evaluation of municipal solid waste composting and potential compost markets. Bioresource Technology, 100: 4301- 4310.

Gueu S, Yao B, Adouby K, Ado G. 2006. Heavy Metals Removal in Aqueous
Slution by Activated Carbons Prepared from Coconut Shell and Shell of Palm Tree. Journal of Applied Sciences, 6(13): 2789-2793.

Hargreaves JC, Adl MS, Warman PR. 2008. A review of the use of composted municipal solid waste in agriculture. Agriculture, Ecosystems and Environment, 12: 31-14.

IFE (Institut français de l'environnement). 2006. Rapport, le sol et le sous-sol. www.ifen.fr/acces-thermatique/sol/le-sol/ la-pollution-diffuse-des-sols-par-leselements traces.html.

Koffi LCK, Adouby K, Wandan EN, Yao B, Kotchi KP. 2010. Sorption and desorption of $\mathrm{Pb}$ (II) from aqueous solution using Triplochiton scleroxylon sawdust as sorbent. J. Applied Sci., 10: 1536-1544.

Lemière B, Seguin JJ, Le Guern C. 2008. Guide sur le comportement des polluants dans les sols et les nappes. Document du BRGM 300.

Mathieu C, Pieltain F. 2003. Analyse Chimique des Sols/Méthodes Choisies. Editions TEC et DOC : Paris.

Tumuhairwe JB, Tenywa JS, Otabbong E, Ledin S. 2009. Comparison of four lowtechnology composting methods for market crop wastes. Waste Management. 29: 2274-2281.

Waksman SA. 1936. Humus, Origin, Chemical Composition and Importance in Nature, Baillère. Tindall and Cox: London.

Yobouet YA, Adouby K, Trokourey A, Yao B. 2010. Cadmium, Copper, Lead and Zinc speciation in contaminated soils. International Journal of Engineering Science and Technology, 2(5): 802-812. 